\title{
Impactos da falta de comunicação entre sistemas de informação em uma empresa do setor metalomecânico: um estudo de caso
}

\author{
Impacts of the lack of communication between information systems in a company in the \\ metalworking sector: a case study
}

Impactos de la falta de comunicación entre sistemas de información en una empresa del sector metalmecánico: un caso de estúdio

Recebido: 29/12/2020 | Revisado: 29/12/2020 | Aceito: 06/01/2021 | Publicado: 07/01/2021

\author{
Maicon Rafael Hammes \\ ORCID: https://orcid.org/0000-0001-6864-3887 \\ Faculdade Horizontina, Brasil \\ E-mail: maiconhammes@gmail.com \\ Argemiro Luis Brum \\ ORCID: https://orcid.org/0000-0002-8763-9514 \\ Universidade Regional do Noroeste do Estado do Rio Grande do Sul, Brasil \\ E-mail: argelbrum@unijui.edu.br \\ Donizete Mulling Franck \\ ORCID: https://orcid.org/0000-0002-2231-2756 \\ Faculdade Horizontina, Brasil \\ E-mail: df003675@fahor.com.br \\ Maria Margarete Baccin Brizolla \\ ORCID: https://orcid.org/0000-0002-5120-0729 \\ Universidade Regional do Noroeste do Estado do Rio Grande do Sul, Brasil \\ E-mail: marga.brizolla@unijui.edu.br \\ Marcela Hammes Teixeira \\ ORCID: https://orcid.org/0000-0001-6662-384X \\ Universidade Federal do Rio Grande do Sul, Brasil \\ E-mail: marcelahteixeira@gmail.com
}

\begin{abstract}
Resumo
Dentre as ferramentas de apoio à gestão, o sistema de informação tem ocupado um lugar de destaque nas associações, sendo a sincronização de dados e informações de forma automática e em tempo real, relevante dentro deste contexto. Diante disto, o objetivo desta investigação é identificar como proveniente da falta de comunicação entre o Enterprise Resource Planning (ERP), responsável por dar suporte ao Planejamento e Controle da Produção (PCP) e o Sistema de Gestão da Produção (SGP), em uma empresa metalomecânica. Para realizar o trabalho foi realizado um estudo de caso, com abordagem exploratória e descritiva. Os resultados indicam que a falta de comunicação entre sistemas acarreta redução do índice de disponibilidade do equipamento em 2,11\%. Considerando os desperdícios devido a um apontamento de ordem, somados custos com hora-máquina e hora-homem, estima-se que o valor que a empresa deixa de arrecadar margeia de $\mathrm{R} \$ 14.000,00$ por ano no equipamento analisado.
\end{abstract}

Palavras-chave: Sistemas de informação; Tecnologias de informação; Disponibilidade.

\begin{abstract}
Among the management support tools, the information system has occupied a prominent place in organizations, with the synchronization of data and information automatically and in real time, relevant within this context. Given this, the objective of this investigation is to identify the implications arising from the lack of communication between the Enterprise Resource Planning (ERP), responsible for supporting Production Planning and Control (PCP) and the Production Management System (SGP), in a metalworking company. To carry out the work, a case study was carried out, with an exploratory and descriptive approach. The results indicate that the lack of communication between systems leads to a reduction in the equipment availability index by $2.11 \%$. Considering the waste due to ordering, plus costs with machine hour and man hour, it is estimated that the value that the company fails to collect borders the R \$ $14,000.00$ per year in the analyzed equipment.
\end{abstract}

Keywords: Information systems; Information technologies; Availability.

\section{Resumen}

Entre las herramientas de apoyo a la gestión, el sistema de información ha ocupado un lugar destacado en las organizaciones, con la sincronización de datos e información de forma automática y en tiempo real, relevante en este 
contexto. Ante esto, el objetivo de esta investigación es identificar las implicaciones derivadas de la falta de comunicación entre el Planificación de Recursos Empresariales (ERP), responsable de dar soporte a la Planificación y Control de la Producción (PCP) y el Sistema de Gestión de la Producción (SGP), en una empresa metalmecánica. Para la realización del trabajo se realizó un estudio de caso, con un enfoque exploratorio y descriptivo. Los resultados indican que la falta de comunicación entre sistemas conduce a una reducción del índice de disponibilidad de equipos en un $2,11 \%$. Considerando el desperdicio por pedido, más costos con hora máquina y hora hombre, se estima que el valor que la empresa no recolecta bordea los R \$14.000,00 anuales en los equipos analizados.

Palabras clave: Sistemas de información; Tecnologías de la información; Disponibilidad.

\section{Introdução}

Sistemas e tecnologias de informação (TI), são componentes essenciais de negócios e organização bem-sucedidas (O’brien, 2013). A informação é fundamental para as empresas adquirirem uma postura competitiva quando expostas a um mercado comum. Fatores como competitividade, produtividade e qualidade não podem ser almejados sem a existência da informação. Tecnologia da informação é todo e qualquer dispositivo que trate e/ou processe dados e/ou informações, de forma sistêmica ou esporádica, quer esteja aplicado no produto ou processo (Cruz, 2014).

As TIs, incluindo sistemas com base na internet, têm hoje papel vital e crescente na administração, sendo capazes de auxiliar todos os tipos de negócios, melhorando índices de produtividade e eficácia de seus processos, e a tomada de decisão gerencial. Os sistemas e as tecnologias da informação são indispensáveis para a competitividade o sucesso dos negócios no ambiente global dinâmico e de rápidas mudanças (O’brien, 2013).

Os sistemas de informações (SI), oferecem recursos para a geração, tratamento e armazenamento de dados e informações. Estão cada vez mais sofisticados, complexos e completos, trazendo mudanças nos processos, na estrutura e estratégia das empresas. Empresas que buscam competitividade precisam fazer uso dessas ferramentas, visando melhoria contínua e aperfeiçoamento de todos os processos em suas áreas de atuação (Stair, 2015).

Maia, Mendes e Souza (2015) apresentam um estudo sobre a importância da interligação entre o Sistema de Informações sobre Mortalidade e Sistema de Informações sobre nascidos vivos em cinco cidades brasileiras. O resultado foi a qualificação das estatísticas e a possibilidade de aplicação de melhorias no sistema de vigilância de saúde. Albertin e Yoshikumi (2018) abordam um estudo sobre a importância do sistema de informação estratégico para o planejamento e enfrentamento de desafios ambientais. Barbosa et al (2015) expuseram uma pesquisa na qual a ausência de interligação entre o sistema de informação para atividade logística e o ERP não atende a necessidade de $80 \%$ dos gestores. Fialho, Gonzalez Junior e Santos (2016) publicaram um artigo de análise de desempenho de sistemas implantados em empresas do comércio varejista que constatou a importância da amplitude de encaixe dos sistemas e a qualidade do processo de implantação. Pinochet (2016) disserta sobre o uso de sistemas de informação na indústria de alimentos como um fator estratégico. O estudo concluiu que a maturidade dos negócios precisa estar nivelada para a integração de processos para que a empresa tenha sucesso.

Portanto, a tecnologia da informação contribui através da infraestrutura e dos sistemas de informação para gestão das organizações a partir da disponibilidade em tempo real de dados que são utilizados no processo de tomada de decisão (Pinochet, 2016; Fialho, Gonzalez Junior \& Santos, 2016).

Neste contexto, busca-se saber que implicações decorrem da falta de comunicação entre o Enterprise Resource Planning (ERP), responsável por dar suporte ao Planejamento e Controle da Produção (PCP) e o Sistema de Gestão da Produção (SGP), em uma empresa metalomecânica? Para tanto o objetivo desta investigação é identificar as implicações provenientes da falta de comunicação entre os referidos sistemas.

Com a globalização e o desenvolvimento de novas tecnologias, obrigatoriedade de envio de informações tributárias em tempo real e a necessidade de melhorar os processos produtivos em busca de diferencial competitivo, os sistemas de informação vem evoluindo nos últimos anos. Em alguns casos, as empresas utilizam mais de um sistema de diferentes 
fabricantes. Justifica-se assim o estudo com o mapeamento da fração de tempo gasta pelo colaborador para que se façam, de forma manual, os devidos registros antes do início e após o término da Ordem de Produção (OP), denominado Apontamento de Ordem no sistema que gerencia o PCP. Gerando desta forma ociosidade nas máquinas de usinagem Computer Numeric Control (CNC), impactando diretamente no indicador de disponibilidade do mesmo e consequentemente aumentando custos de produção e reduzindo receitas.

\section{Referencial Teórico}

A fim de aprofundar o entendimento acerca da temática proposta para o estudo, visando responder a questão de investigação, faz-se necessário o embasamento teórico. Sendo assim, são abordados os seguintes temas: sistemas de informação, tecnologia da informação e sistemas de informação nos processos produtivos.

\subsection{Sistemas de informação}

A partir do momento em que surgiram as primeiras organizações o homem vem desenvolvendo a tecnologia, seja ela na forma de técnicas, seja na forma de sistemas, métodos e equipamentos, a fim de cumprir de forma mais completa as suas atribuições. Um sistema pode ser compreendido como um conjunto de partes interagentes e interdependentes, formando um todo com determinado objetivo e função, logicamente estruturadas e organizadas para sua finalidade (Oliveira, 2005; Cassarro, 2010).

A empresa em si é uma estrutura estática, movimentada pelo conjunto de sistemas de informação, de modo que sejam possíveis o planejamento, a organização, a coordenação e o controle das operações. Sendo assim, os sistemas de informações compõem os maiores e mais valiosos ativos da empresa, e cada uma das áreas de atividade pode ser considerada um subsistema. A informação inserida no contexto empresarial tem como propósito habilitá-la a alcançar seus objetivos através do uso eficiente dos recursos, sendo eles pessoas, materiais, equipamentos, tecnologia e capital (Oliveira, 2005; Cassarro, 2010).

Sistemas possuem entrada de dados (input), processamento e saída das informações (output) e feedback (Figura 1). Teoricamente, existem dois tipos de sistema: o aberto, o qual acaba sofrendo influência do meio e ao mesmo tempo influencia o meio; e o fechado, o qual não sofre influência alguma e nem provoca alterações no meio com suas ações internas (Rosini, 2012).

Figura 1. Esquema teórico de qualquer sistema.

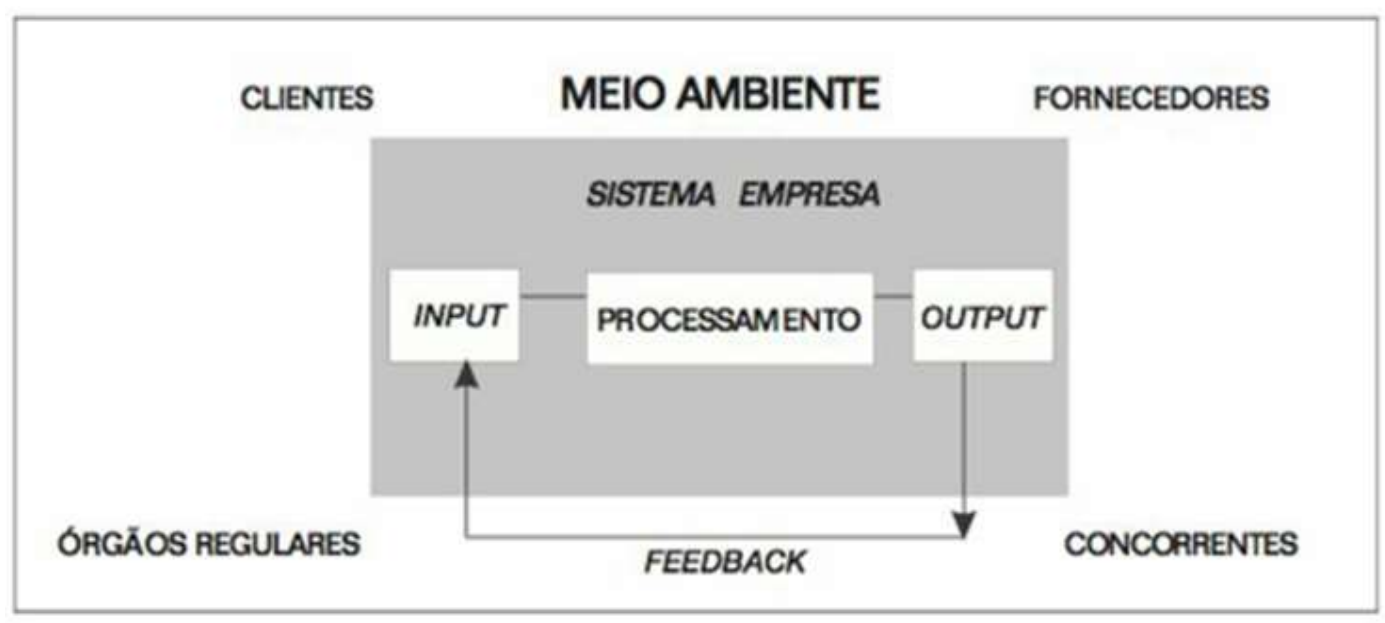

Fonte: Rosini (2012). 
Para Cassarro (2010), as informações dentro de uma empresa podem ser classificadas referentes ao seu propósito em dois grupos:

- informação operativa: é necessária para realização de uma função e, ou operação;

- informação gerencial: consiste em um resumo de informações operativas que chega até o gerente para que este fique a par da situação, de sua responsabilidade, a fim de que possa tomar decisões.

Qualquer informação que seja produzida na empresa apresenta uma série de características e atributos técnicos, sendo que estas dizem respeito a custos de produção versus benefício, oportunidade, relevância e por fim comparação em tendência (Cassarro, 2010).

\subsection{Tecnologia da informação}

A tecnologia da informação pode ser compreendida como recursos tecnológicos e computacionais para armazenar e gerar o uso da informação e do conhecimento, sendo conectados entre si ou standalone (Cruz, 2014). Fundamentada nos seguintes componentes: hardware que contempla os computadores e seus respectivos dispositivos e periféricos; software que contempla os programas nos seus diversos tipos, como o software operacional, de redes, aplicativos, utilitários e de automação, são responsáveis por dirigir, organizar e controlar os recursos de hardware, fornecendo instruções, comandos. Os sistemas de telecomunicação interligam hardware e software. As comunicações de dados são um subconjunto especializado de telecomunicações que se referem à coleta, processamento e distribuição eletrônica de dados, entre os dispositivos de hardware de computadores (Stair, 2015).

Já o banco de dados é um conjunto de elementos relacionados, e logicamente integrados, que consolida registros anteriormente fornecidos em arquivos separados em um lote comum de dados, que fornece dados para várias aplicações. São independentes dos programas de aplicação que os usam e do tipo de dispositivos de armazenamento. Desta forma, os bancos de dados contêm elementos de dados que descrevem entidades e as relações entre estas entidades (O’brien, 2013).

A informação de rápido acesso é fundamental para a administração eficaz em um mercado aberto e comum. Entender como a tecnologia da informação interage é um aspecto importante da etapa de avaliação. É a mensuração das mesmas, quanto a sua viabilidade na organização e para os processos envolvidos. Quando a organização opta por adotar uma nova tecnologia, é feita uma estimativa da relação custo-benefício, comparando indicadores de qualidade e produtividade. A partir da análise de oportunidades e ameaças externas, pontos fortes e fracos internos, são identificadas as vantagens competitivas existentes no contexto que a empresa se apresenta, e seus potenciais. O processo de planejamento nesse ponto apresenta diversos desdobramentos paralelos, como marketing, produção e tecnologia (Rosini, 2012).

Os sistemas de informações cada vez apresentam mais recursos, com o propósito fundamental de constituir um sistema integrado. Os sistemas ERP permitem que as decisões e os bancos de dados de todas as partes da organização sejam integrados, sendo o equivalente ao sistema nervoso central da organização, detectando as informações sobre as condições de diferentes partes da empresa e as retransmitindo a outras partes da empresa que necessitam delas, com informações atualizadas em tempo real (Rosini, 2012; Slack, 2015).

Com a integração dos processos organizacionais, desenvolveu-se a ideia de criar sistemas transacionais de informações, que sejam constituídos de módulos parametrizados e personalizados para a realidade de cada uma das empresas, integrados entre si, a fim de atender a empresa como um todo. O Materials Resource Planning, (MRP) evoluiu para o ERP, desenvolvido com o objetivo de integrar sistemas de escritório com a fábrica, aplicando o que é chamado de as melhores práticas de administração de negócios (Rosini, 2012). 
Entre os benefícios oferecidos por sistemas ERP, pode-se citar: a padronização de dados, o que torna os dados mais transparentes, facilita comparação de resultados e ressalta a exposição de anomalias da informação, o que facilita a utilização de diferentes indicadores de resultados na empresa; a padronização de processos, que consiste na redução e otimização de processos, promovendo mudanças em formas de trabalho; a mudança contínua da organização, seguindo uma política de mudanças contínuas e planejadas para permitir aprendizagem organizacional para a eficiência (Rosini, 2012).

\subsection{Sistemas de informação nos processos produtivos}

O sistema de informação gerencial, empregado de maneira correta, pode proporcionar inúmeros benefícios para a empresa. Dentro deste contexto destacam-se: a redução dos custos operacionais; melhoria no acesso à informação e relatórios mais assertivos; aumento de produtividade; maior interação entre os tomadores de decisões; redução do grau de centralização das decisões; aumento dos níveis de motivação pessoas envolvidas; e redução de funcionários em atividades burocráticas (Oliveira, 2005).

Sistemas ERP são utilizados em indústrias, instituições de ensino, organizações de serviço profissional, varejistas e organizações de assistência à saúde, com diversas necessidades de negócios. Desta forma, os anseios de uma organização multinacional são muito diferentes das de uma pequena empresa. Assim, não existe nenhum software ERP de um único fornecedor melhor para todas as organizações (Stair, 2015).

No chão de fábrica, a tecnologia da informação abrange equipamentos de manufatura, movimentação de materiais, desenhos (processos assistidos por computador), planejamento, controle e gestão. Nos setores administrativos inclui-se o processamento de textos, arquivamento automático, processamento de transações, conferências, planilhas, correio e quadros eletrônicos, pesquisa em banco de dados, e sistemas de suporte a decisões. As implementações de tecnologias da informação vão desde ilhas de automação, ou seja, aplicação de tecnologias isoladas, à sistemas mais integrados e complexos de manufatura, que interligam as atividades de desenho, manufatura, movimentação de materiais e planejamento de controle (Rosini, 2012).

Stair (2015) complementa que implantar o ERP traz benefícios que abrangem o acesso aos dados com mais qualidade para a tomada de decisão operacional, eliminação de sistemas ineficientes ou obsoletos e padronização da tecnologia. Esses benefícios estão muitas vezes vinculados aos fornecedores de ERPs, que desenvolveram sistemas especializados, oferecendo soluções eficazes para setores específicos da atividade e segmentos de mercado.

A TI (Tecnologia da Informação) é o tipo mais comum de tecnologia nas operações e inclui qualquer dispositivo capaz de coletar, manipular, estocar e/ou distribuir a informação. A função do PCP é antecipar o processo produtivo desenvolvendo uma programação que garanta maior racionalidade e atenda as limitações de capacidade produtiva dos equipamentos e recursos humanos envolvidos (Slack, 2015; Rocha, 1995).

O PCP é o setor de apoio responsável pela orientação, coordenação e aplicação dos recursos produtivos de forma a atender os planos estabelecidos desde o estratégico até o operacional, o mais próximo das metas estabelecidas. Para atingir tais objetivos, o PCP processa dados vindos de diversas áreas do sistema produtivo, transformando-os em informações úteis para toda a cadeia produtiva (Chiavenato, 2005).

Também promove o gerenciamento de grande volume de informações, oriundas dos mais diversos setores da empresa industrial, sendo fundamental que todas as informações relevantes, que estão espalhadas em toda a organização, estejam reunidas. Assim, as decisões de quando as atividades devem ocorrer, onde, quem deve executá-las, quanta capacidade será necessária para executá-las, serão mais assertivas (Rocha, 1995; Slack, 2015). 
O planejamento realizado pelo PCP precisa chegar à execução, as informações são levadas ao setor produtivo pela Ordem de Produção (OP), que contém elementos do planejamento como quantidade a que será produzida, os recursos a serem utilizados e propicia que as informações geradas na fase de execução sejam registradas. Essas, geralmente são informatizadas, onde através de computadores colocados em pontos estratégicos acontece o lançamento das informações, dando assim condições de controlar o desempenho do setor e avaliar as pessoas envolvidas no trabalho (Rocha, 1995).

O setor de PCP necessita conhecer a situação de ocupação e disponibilidade dos equipamentos, assim como identificar qual OP está sendo realizada em cada máquina, com acompanhamento criterioso das perdas, sejam elas de matéria-prima, hora-máquina, hora-homem ou de produtos. O controle deve ser feito a fim de possibilitar o acompanhamento permanente do percentual de desperdícios ocorrido no processo (Rocha, 1995). Dentro deste contexto, a disponibilidade pode ser entendida como a relação entre o tempo que o equipamento ficou disponível para produzir, e o tempo total, ou seja, o tempo que poderia ficar disponível para a operação (Kardec \& Nascif, 2001).

\section{Metodologia}

O estudo objetiva identificar as implicações provenientes da falta de comunicação entre o Enterprise Resource Planning (ERP), responsável por dar suporte ao Planejamento e Controle da Produção (PCP) e o Sistema de Gestão da Produção (SGP), em uma empresa metalomecânica. No intuito de atender a proposta, foi realizado uma pesquisa descritiva, pois visa descobrir a existência de associações entre variáveis, e vai além da simples identificação, pretendendo determinar a natureza dessa relação (Gil, 2019).

Quanto aos procedimentos tem-se uma pesquisa documental, efetuada a partir dos documentos disponibilizados pela organização objeto desta investigação, de acordo com o apregoado por Gil (2019). Ainda, trata-se de um estudo de caso, de caráter empírico, que investiga um fenômeno atual dentro do contexto da vida real, geralmente considerando que as fronteiras entre o fenômeno e o contexto não estão claramente definidas (Yin, 2001). Foi realizada uma investigação aprofundada em uma indústria metalmecânica de porte médio, localizada na região Noroeste do RS.

O estudo é de abordagem qualitativa, uma vez que usa como estratégias de investigação o estudo empírico baseado na teoria (Creswell, 2007). Este estudo tem como preocupação central descrições, compreensões e interpretações dos fatos ao invés de medições (Martins \& Theóphilo, 2007), também por se tratar de estudo de caso e este é um elemento essencial das pesquisas qualitativas.

O objeto de estudo foi o setor de usinagem industrial de uma empresa do ramo metalmecânico, a qual fabrica diversas peças e componentes utilizadas em tratores, colheitadeiras, plantadeiras, armazenagem de grãos e implementos agrícolas em geral. O setor utiliza predominantemente tornos mecânicos CNCs, que são máquina utilizada na indústria com o objetivo de remover material de forma programável e precisa, oferecendo componentes sem variação nas medidas em relação ao seu projeto.

Como instrumentos de pesquisa foram utilizados os documentos disponibilizados e a observação direta, os quais possibilitaram a obtenção de dados referentes ao PCP do setor de usinagem, envolvendo os sistemas de informação utilizados, que é o ERP e o SGP. Para tanto, optou-se pela coleta de dados feito in loco. Os dados foram extraídos de gráficos e relatórios gerados pelo SGP, utilizados para mensurar o desempenho de cada equipamento, em razão da ausência de comunicação entre os dois sistemas, sendo que um atua como complemento do outro. Também foi feito uso da entrevista despadronizada, para aprofundar ou esclarecer questões que surgiram durante a coleta dos dados.

Com o intuito de atender o objetivo proposto, de modo a responder a questão de investigação, buscou-se: compreender a importância dos Sistema de Informação dentro de uma empresa, a luz da teoria a respeito do tema; identificar as 
ferramentas de TI utilizadas para o desenvolvimento das atividades de PCP e acompanhamento da produção; e analisar as implicações gerados nos indicadores de disponibilidade dos equipamentos e os custos que estes representam.

As técnicas de análise de dados utilizadas foram a análise documental e de conteúdo (Bardin, 1977), sendo que a partir dos dados coletados do SGP, realizou-se o acompanhamento em tempo real dos processos de produção, envolvendo mão de obra, matéria prima, máquinas e demais recurso necessários a produção. Este sistema armazena dados referentes ao histórico de cada equipamento, o que possibilitou, diagnosticar o desenvolvimento e a evolução ao longo do tempo de cada equipamento no setor produtivo.

\section{Resultados e Discussões}

Nesta parte são apresentadas as análises e interpretações dos dados, necessários a responder a questão de investigação, no que diz respeito aos sistemas de informação utilizados para planejamento e monitoramento da produção.

Para dar suporte à programação e ao planejamento da produção, a empresa conta com o auxílio de um sistema ERP, responsável por gerenciar as necessidades de produção, contudo as OPs são geradas posteriormente a uma análise criteriosa, feita item a item pelos responsáveis do setor. Depois de geradas no sistema ERP, e impressas as OPs, é necessário que elas sejam cadastradas manualmente no SGP antes de serem disponibilizadas ao setor produtivo.

O SGP é um sistema integrado de software e hardware que proporciona a coleta de dados industriais e gestão em tempo real dos processos de manufatura. Esses dados extraídos diretamente dos centros de usinagem e tornos mecânicos do tipo CNCs, processados, geram indicadores que são apresentados em forma de gráficos, quadros interativos e relatórios atualizados automaticamente e instantaneamente.

Ambos os sistemas de informações utilizados partilham em parte dos mesmos dados e informações, porém, a forma com que as utilizam e os resultados apresentados é que diferem. O SGP necessita ser constantemente alimentado com as informações geradas nas OPs. Para que este tenha um desempenho esperado é necessário que toda OP seja cadastrada, quanto ao seu número de registro, código do item a ser produzido e a quantidade do lote.

Sempre que o operador de algum determinado equipamento CNC iniciar a produção de uma nova OP, será necessário que se desloque até o posto avançado com suporte do sistema ERP para que dê o start inicial daquela operação. Todo este processo é feito fora do posto de trabalho. No posto avançado é feita a leitura de um código de barras, que identifica a operação que será realizada. Em seguida o operador informa seu código de cadastro, para que se tenha o registro do funcionário que realizou a operação, a fim de possibilitar a rastreabilidade do processo dentro do sistema ERP.

Processo semelhante é realizado no SGP, porém, o operador não precisa sair do seu posto de trabalho. Todas as informações referentes ao número da OP e a operação realizada, são lançadas em um equipamento acoplado na máquina CNC.

Quando determinada OP tem uma etapa concluída, o operador se desloca até o posto avançado para fazer o fechamento do processo na OP, novamente fazendo a leitura do código de barras que identifica a etapa concluída, informando a quantidade produzida, diferenciando peças conformes de peças não conformes e, por fim, informando seu código pessoal de cadastro. É importante salientar que no chão de fábrica apenas o mesmo colaborador que iniciou a operação consegue fazer o seu fechamento.

No que diz respeito ao SGP, o fechamento da OP ocorre de forma automática, assim que a quantidade registrada no sistema for atingida. Quanto às peças não conformes, cabe ao operador informar a causa do problema no momento exato que identifica o item desconforme saindo do equipamento, facilitando o processo de rastreabilidade.

Toda e qualquer parada que vir a acontecer durante o espaço de tempo programado para produzir é registrada pelo Sistema de Gestão da Produção. Paradas inferiores a um minuto, o registro é feito automático, como micro parada. Quando o 
tempo é superior a este é necessário que a operadora informe ao sistema o motivo da parada, para que a máquina volte a operar normalmente. Toda vez em que o operador se desloca de seu posto de trabalho para o posto avançado, a fim de realizar os devidos registros de OP no sistema ERP, gera-se uma parada onde o operador informa o motivo. Este fica cadastrado como Apontamento de Ordem, sendo o tempo em que acontece a transferência manual de informações do setor produtivo da fábrica para o sistema ERP.

Dentro da realidade da empresa, o PCP é responsável por gerenciar e coordenar a produção de grande quantidade de produtos ao longo do mês, visando atender demanda existente. Simultaneamente, a gestão da produção nestes moldes, visa à redução de capital imobilizado na forma de estoque de produtos manufaturados.

Partindo do pressuposto de que inúmeros itens são produzidos em diferentes momentos no decorrer do mês, gera-se assim um acumulado significativo de horas desperdiçadas apenas com o Apontamento de Ordens, ou seja, transferência de forma manual das informações produzidas, coletadas e processadas automaticamente pelo SGP para o sistema ERP. Paradas para Apontamento de Ordem é o resultado direto da falta de comunicação entre os dois Sistemas de Informação, e impactam diretamente no indicador de disponibilidade dos equipamentos.

A partir das informações fornecidas pelo SGP, é possível visualizar a dinâmica dos processos de manufatura. As informações operativas têm a finalidade de acompanhar o andamento das atividades, se estão sendo executados conforme o planejado, e se necessário, alinhar os processos em tempo real, para atingir as metas do setor de PCP.

O Sistema de Gestão da Produção traz uma relação completa de todo o tempo desperdiçado, ou seja, tempo em que os equipamentos poderiam estar agregando valor à matéria prima, porém, por algum motivo não o estão realizando. Para mais fácil compreensão, estas informações podem ser apresentadas na forma de tabelas, tratando cada equipamento de forma individual (Quadro 1). Sendo ainda possível identificar o momento exato em que as paradas acontecem através da análise de relatórios disponibilizados pelo sistema.

Tabela 1: Motivos de paradas do equipamento Z.

\begin{tabular}{c|l|l|c|c}
\hline Código & \multicolumn{1}{|c|}{ Tipo de Parada } & \multicolumn{1}{c|}{ Descrição } & Tempo (h) & Qtde Paradas \\
\hline 240 & Não relativa ao recurso & Op. Em outra Maq. & 25,47 & 11 \\
\hline 60 & Setup & Setup Preparação & 11,47 & 43 \\
\hline 90 & Relativa ao Recurso & Amostra & 7,07 & 2 \\
\hline 80 & Relativa ao Recurso & Retrabalho & 6,75 & 29 \\
\hline 61 & Setup & Setup Peça 1 & 6,62 & 19 \\
\hline 5 & Relativa ao Recurso & Apontamento de Ordem & 6,61 & 51 \\
\hline 10 & Manutenção & Máquina em manutenção & 6,49 & 7 \\
\hline 40 & Não Relativa ao Recurso & Troca de ferramenta & 5,37 & 83 \\
\hline 130 & Não Relativa ao Recurso & Limpeza & 5,33 & 33 \\
\hline \multicolumn{2}{r}{} & Outras Total: & 16,46 & 205 \\
\hline \multicolumn{2}{r}{} \\
\multicolumn{2}{r}{}
\end{tabular}

Fonte: Sistema de Gestão da Produção.

No período de $1^{\circ}$ de março até 31 de março de 2020 o equipamento $Z$ em questão, apresentou um total de 97,64 horas paradas, isto considerando todos os tipos possíveis que impactam diretamente no indicador de disponibilidade. Neste período, foram realizados 51 Apontamentos de Ordem, que representam 6,61 horas, ou seja, 6,45\% do tempo total que o equipamento ficou inoperante.

Nos últimos 12 meses o equipamento $\mathrm{Z}$ operou em dois turnos, totalizando 3.013,99 horas analisadas. Destas foram contabilizadas 1.620,61 horas em produção e 1.393,37 horas paradas (quadro 2). Do montante de horas paradas, 63,64 horas são provenientes do Apontamento de Ordem, que somaram 542 registros. 
Tabela 2. Motivos de paradas por recuso.

\begin{tabular}{c|l|l|c|c}
\hline Código & \multicolumn{1}{|c|}{ Tipo de Parada } & \multicolumn{1}{c|}{ Descrição } & Tempo (h) & Qtde Paradas \\
\hline 240 & Não relativa ao recurso & Op. Em outra Maq. & 275,51 & 184 \\
\hline 60 & Setup & Setup Preparação & 253,97 & 703 \\
\hline 10 & Manutenção & Máquina em manutenção & 147,63 & 177 \\
\hline 61 & Setup & Setup Peça 1 & 106,86 & 403 \\
\hline 90 & Relativa ao Recurso & Amostra & 102,35 & 50 \\
\hline 40 & Não relativa ao Recurso & Troca de ferramenta & 79,41 & 754 \\
\hline 5 & Relativa ao Recurso & Apontamento de ordem & 63,64 & 542 \\
\hline 62 & Setup & Medição Setup & 55,52 & 254 \\
\hline 80 & Relativa ao Recurso & Retrabalho & 55,19 & 386 \\
\hline 120 & Relativa ao Recurso & Ajuste do software & 206,96 & 170 \\
\hline & Outras & $1.393,37$ & 3050 \\
\hline
\end{tabular}

Fonte: Sistema de Gestão da Produção.

$\mathrm{O}$ indicador de disponibilidade do equipamento $\mathrm{Z}$ para o período, que representa relação entre o tempo que o equipamento ficou disponível para produzir e o tempo total programado para produzir, ficou em 53,77\%. A falta de comunicação entre o sistema ERP e o SGP, mensurada pelo critério Apontamento de Ordem, representou, nos últimos 12 meses, $2,11 \%$ de perdas no índice de disponibilidade do equipamento estudado.

O problema em questão pode ser solucionado através da interação entre o sistema ERP e o SGP, fazendo com que dados e informações geradas no sistema ERP, com apoio do setor de PCP, sejam transferidas automaticamente para o sistema SGP. Da mesma forma a interação deve ocorrer no sentido inverso, ou seja, dados e informações geradas pelo equipamento CNC, trabalhadas e processadas pelo SGP, devem ser transferidas de forma automática a fim de retroalimentar o sistema ERP.

A comunicação ocorrendo de forma automática, instantânea e contínua traz inúmeros benefícios, tanto para o setor de PCP quanto para todos aqueles envolvidos diretamente na manufatura dos produtos. Para o setor de PCP, o fácil acesso às informações, pois se encontram todas reunidas e interagindo em um só local, permite que a utilização de planilhas intermediárias seja eliminada.

Para os colaboradores responsáveis por operar as máquinas de usinagem CNC, a interação entre os sistemas resulta na dispensa do deslocamento para o posto avançado ERP. Reduzir a movimentação diária do colaborador é um fator importante, uma vez que melhora a qualidade de vida do colaborador durante sua jornada de trabalho.

Para a empresa, os benefícios estão relacionados com a redução de desperdícios no processo de manufatura e, consequentemente, com o aumento da receita. Ainda, a melhor utilização dos recursos humanos, uma vez que a parcela de tempo desperdiçada com o Apontamento de Ordem é equivalente a, aproximadamente, oito dias de trabalho de um colaborador no período de um ano.

$\mathrm{O}$ equipamento $\mathrm{Z}$, objeto deste estudo, possui um valor de mercado aproximado de $\mathrm{R} \$ 300.000,00$. Isso considerado todos os fatores que geram depreciação do equipamento no decorrer do tempo de utilização dentro da empresa. O setor de usinagem conta, no seu total, com nove CNCs. Estes, em sua maioria, com valor de mercado superior ao equipamento Z.

As implicações de os equipamentos CNCs estarem parados, em período programado para operar, vão além da redução dos indicadores de disponibilidade e das receitas, afetam a confiabilidade que os clientes atribuem à empresa. Todo tempo em que o equipamento deixa de realizar o processo de manufatura da matéria prima, dentro de um período programado, aumenta as chances de o produto não ser entregue na data requerida pelo cliente. 


\section{Considerações Finais}

O objetivo básico da informação é habilitar a empresa a alcançar seus objetivos e metas pelo uso racional e eficiente dos recursos disponíveis, sendo as informações responsáveis por decidir o presente e o futuro da mesma. A velocidade em que as informações são assimiladas, e o sistema realimentado, resulta na rapidez na tomada de decisões e consequentemente no seu grau de assertividade. Enxugar todos os processos dentro da organização é torná-la mais competitiva no âmbito global, pois produzir mais com menos recursos utilizados é um desafio que deve ser almejado cada dia.

No que se refere ao objetivo do estudo que é analisar as implicações provenientes da falta de comunicação entre o Enterprise Resource Planning (ERP), responsável por dar suporte ao Planejamento e Controle da Produção (PCP) e o Sistema de Gestão da Produção (SGP), em uma empresa metalomecânica, entende-se que foi atingido, sendo apresentados os efeitos negativos no índice de disponibilidade do equipamento. A partir da análise do histórico de produção e de paradas da máquina foi identificado que houve uma queda no índice disponibilidade do equipamento, ocasionado pelo Apontamento de Ordem.

$\mathrm{O}$ equipamento $\mathrm{CNC}$ analisado possui o valor monetário hora-máquina trabalhada em torno de $\mathrm{R} \$ 200,00$ reais. Considerando as horas desperdiçadas, devido a Apontamento de Ordem, acrescidas do custo hora-homem para a empresa, estima-se que a empresa deixa de arrecadar cerca de $\mathrm{R} \$ 14.000,00$. Este valor representa as perdas de um único equipamento ao longo do ano, lembrando que a empresa conta com mais $10 \mathrm{CNCs}$ similares.

Sugere-se que novos estudos sejam realizados na área, considerando outros equipamentos CNCs do setor de usinagem, assim como maior quantidade de equipamentos no setor. É importante levar em conta as particularidades que envolvem cada situação, a fim de apresentar aos gestores e proprietários os benefícios envolvendo a confiabilidade das informações transferidas de um sistema para outro, além dos ganhos com as reduções de desperdícios ocasionados pela falta de comunicação de tais Tecnologias da Informação.

\section{Referências}

Albertini, A. L., Yoshikuni, A. C. Sistemas de Informação Estratégicos Habilitando Estratégia-como-prática na Incerteza Ambiental. Revista RAC. 22(4), 552576.

Barbosa, et al. A contribuição dos sistemas de informação para logística reversa: uma pesquisa (survey). Revista Eletrônica Gestão \& Saúde. 6(2), 1108-1124.

Bardin, L. (1997) Análise de conteúdo. Edições 70.

Cassaro, A. C. (2010) Sistemas de informação para tomada de decisões. (4a ed.), Cengage Learning.

Chiavenato, I. (2005) Administração dos materiais: uma abordagem introdutória. Elsevier.

Creswell, J. W. (2007). Projeto de pesquisa: métodos qualitativo, quantitativo e misto. (2a ed.), Artmed.

Cruz, T. (2014) Sistemas de informações gerenciais: tecnologias da informação e as organizações do século XXI. (4a ed.), Atlas.

Fialho, S. H., Gonzalez Junior, I. P. \& Santos, E. M. Avaliação dos sistemas de informação nas organizações: um estudo de caso em empresas do comércio varejista da cidade de Cruz das Almas - BA. Revista Gestão e Tecnologia. 6(2), 20-36

Gil, A. C. (2019) Métodos e técnicas de pesquisa social. (7a ed.) Atlas.

Kardec, A. P., \& Nascif, J. A. X. (2001) Manutenção: função estratégica. Qualitymark Ed.

Maia, L. T. de S., Mendes, A. C. G., \& Souza, W. V. A contribuição do linkage entre o SIM e SINASC para a melhoria das informações da mortalidade infantil em cinco cidades brasileiras. Revista Brasileira de Saúde Materna Infantil. 15, 57-66.

Martins, G. de A., \&Theophilo, C. R. (2007) Metodologia da investigação científica para ciências sociais aplicadas. Editora Atlas.

O’brien, J. A. (2013) Administração de sistemas de informação. (15a ed.), AMGH.

Oliveira, D. de P. R. (2005) Sistemas de informações gerenciais: estratégicas, táticas, operacionais. (10a ed.), Atlas.

Pinochet, L. H. C. Uso Estratégico dos Sist0emas de Informação no Apoio aos Processos de Negócio: Estudo de Caso na Indústria de Alimentos. Revista Administração em Diálogo. 8(3), 01-25. 
Research, Society and Development, v. 10, n. 1, e18710111572, 2021 (CC BY 4.0) | ISSN 2525-3409 | DOI: http://dx.doi.org/10.33448/rsd-v10i1.11572

Rocha, D. (1995) Fundamentos Técnicos da Produção. Makron Books.

Rosini, A. M. (2012) Administração de sistemas de informação e a gestão do conhecimento. (2a ed.), Cengage Learning.

Slack, N. (2015) Administração da produção. (4a ed.) Atlas.

Stair, R. M. (2015) Princípios de sistemas de informação. Cengage Learning.

Yin, R. K. (2001) Estudo de caso: planejamento e métodos. Bookman. 